\title{
Aminopenicillin allergy
}

\author{
A Romano, D Quaratino, G Papa, M Di Fonso, A Venuti
}

\begin{abstract}
Eighty two children with histories of maculopapular or urticarial rashes during aminopenicillin treatment underwent skin tests, patch tests, radioallergosorbent assays and, in some cases, oral challenges. Hypersensitivity was diagnosed in eight $(9.8 \%)$ : immediate in four with urticarial reactions and delayed (that is cell mediated) in four with maculopapular rashes. In 49 children (38 with maculopapular eruptions, 11 with urticarial/angiooedematous reactions), negative allergologic findings were confirmed using oral challenges with the suspected drug. Maculopapular rashes may reflect delayed hypersensitivity to aminopenicillins, which can be diagnosed on the basis of late intradermal reactions and/or patch test positivity. The allergen panel must include the suspected aminopenicillin itself, as many cases are side chain specific. Most patients with urticarial reactions (more typical of immediate hypersensitivity) will also react to penicilloyl polylysine and minor determinant mixture. The time elapsed between the reaction and testing must be considered if negative results emerge, because IgE mediated sensitivity (unlike cell mediated forms) declines in the absence of antigen exposure.

(Arch Dis Child 1997;76:513-517)
\end{abstract}

Keywords: aminopenicillins; hypersensitivity; maculopapular rash

Many patients who develop rashes, urticaria, or other skin symptoms during $\beta$-lactam treatment are automatically labelled 'allergic to penicillin', and subsequent use of these antibiotics is avoided, even if they appear to be the drugs of choice for treatment of serious infections. Urticaria (or hives), which is one of the classic manifestations of type I (or IgE mediated) hypersensitivity in general, occurs in $4.5 \%$ of patients treated with penicillin or its analogues. ${ }^{1}$ However, many patients who have experienced urticarial reactions to $\beta$-lactams prove to be skin test negative for the major and minor penicillin determinants, and in many cases subsequent administration of penicillin or the suspected drug is well tolerated. ${ }^{2-4}$

Maculopapular or morbilliform rashes are even more common during treatment with these drugs, particularly ampicillin..$^{5-7}$ The incidence of morbilliform rashes during therapy with the latter drug has been estimated at $9.5 \%{ }^{1}$ Until recently, these rashes were thought to be late, idiopathic drug reactions. They usually appear 2-3 days or more after the drug has been started, are not associated with IgE antibodies, and do not appear to predispose the patient to urticarial reactions. ${ }^{1}$ Over the last decade, however, various investigators have come to the conclusion that these rashes often represent type IV (cell mediated) hypersensitivity, which is associated with patch test and/or delayed (that is six or more hours after administration) intradermal test positivity. ${ }^{8-13}$ In some cases, tested skin has been biopsied and found to present histological features similar to those of allergic contact dermatitis, ${ }^{9-13}$ and oral challenges with the suspected drug have provoked delayed onset skin rashes. ${ }^{9} 101213$ This approach has proved to be capable of detecting delayed forms of aminopenicillin hypersensitivity even when several years have elapsed since the last exposure to the drug. ${ }^{12}$ Nevertheless, in many other cases of aminopenicillin associated maculopapular rashes there is no evidence of immune mediated mechanisms of any type, and challenges with the suspected aminopenicillin are well tolerated. ${ }^{12} 14$

The present study was conducted to determine the incidence of true allergy in a group of children with histories of cutaneous eruptions during aminopenicillin treatment.

\section{Patients and methods}

This study included all children with histories of adverse cutaneous reactions to aminopenicillins seen by our staff between January 1990 and December 1995.

The following allergologic investigations were performed.

(1) PRICK AND INTRADERMAL SKIN TESTS

These were done using:

(A) Penicilloyl polylysine (PPL) (Allergopen; Reinbeck, Germany). The undiluted solution was obtained by reconstituting the lyophilised contents of a vial containing 0.175 mg PPL plus $20 \mathrm{mg}$ mannitol in $5 \mathrm{ml}$ buffer. A $1: 10$ dilution of this solution in $0.9 \%$ sodium chloride $(\mathrm{NaCl})$ was initially used. When results were negative, testing was repeated with the undiluted solution.

(B) Minor determinant mixture (MDM) (Allergopen), containing $0.6 \mathrm{mg}$ benzylpenicillin and $0.5 \mathrm{mg}$ benzylpenicilloate $/ \mathrm{ml}$, initially diluted $1: 10000$ in $0.9 \% \mathrm{NaCl}$. Tests were repeated with a 1:10 dilution when initial results were negative.

(C) Potassium penicillin G (Bristol Italiana; Sermoneta, Italy), diluted in $0.9 \% \mathrm{NaCl}$ and administered at increasing concentrations ranging from $0.1 \mathrm{IU} / \mathrm{ml}$ to $10000 \mathrm{IU} / \mathrm{ml}$.

(D) Ampicillin (Amplital, Farmitalia; Milan, Italy) and amoxycillin (Velamox, Zambeletti; 
Table 1 Clinical manifestations described by subjects studied and results of allergologic tests

\begin{tabular}{llll}
\hline Symptoms & $\begin{array}{l}\text { No of patients } \\
\text { studied }\end{array}$ & $\begin{array}{l}\text { No with immediate } \\
\text { hypersensitivity }\end{array}$ & $\begin{array}{l}\text { No with delayed } \\
\text { hypersensitivityt }\end{array}$ \\
\hline Maculopapular rash & 42 & - & 4 \\
Urticaria & 29 & 2 & - \\
$\begin{array}{l}\text { Angio-oedema } \\
\text { Urticaria/angio-oedema }\end{array}$ & 4 & 1 & - \\
$\begin{array}{l}\text { Urticaria/angio-oedema and } \\
\text { dyspnoea }\end{array}$ & 2 & - & - \\
\begin{tabular}{l} 
Erythema \\
\hline
\end{tabular} & 1 & 1 & - \\
\hline
\end{tabular}

* Immediate hypersensitivity diagnosed on the basis of skin tests and RAST.

† Delayed hypersensitivity diagnosed on the basis of delayed intradermal reactivity and patch tests.

Milan, Italy), both used at concentrations of 1 $\mathrm{mg} / \mathrm{ml}$ and $20 \mathrm{mg} / \mathrm{ml}$, after dilution in $0.9 \%$ $\mathrm{NaCl}$.

All haptens were initially tested on the volar forearm skin using the prick technique. Reactions were considered positive when a weal $>3$ $\mathrm{mm}$ in diameter appeared within 15-20 minutes. When negative results were observed, $0.02 \mathrm{ml}$ of the hapten solution was injected intradermally, and readings were made at 20 minutes and 48 hours. Results were defined as positive when weals of $>5 \mathrm{~mm}$ appeared. Positive controls for prick and intradermal tests were performed with histamine $(10 \mathrm{mg} / \mathrm{ml}$ and $1 \mathrm{mg} / \mathrm{ml}$, respectively); normal saline was used as a negative control.

\section{(2) PATCH TESTS}

These were administered with (a) penicillin G (5000 IU/g Vaseline), (b) ampicillin (5\% in Vaseline), and (c) amoxycillin (5\% in Vaseline). The patches were applied to the interscapular region of the back and evaluated according to the criteria of Wilkinson et al after 48 and 72 hours. ${ }^{15}$

(3) RADIOALLERGOSORBENT TESTS (RASTS)

In vitro assays for antigen specific IgE to penicilloyl G, penicilloyl V, ampicillin, and amoxycillin were performed using the Phadebas RAST (Pharmacia; Uppsala, Sweden).

(4) ORAL CHALLENGES

These were performed only in negative cases, on children with histories of either maculopapular reactions or erythematous or urticarial/ angio-oedematous manifestations. The latter had been tested within one year of their last reaction. Informed consent was obtained from all parents, and the children were admitted to the day hospital for 24 hours for administration of the challenge. The drugs - ampicillin (Amplital; Farmitalia) or amoxycillin (Velamox; Zambeletti) - were administered orally at a dose of $5 \mathrm{mg}$. If no reaction occurred within 72 hours, the challenge was repeated one week later with $50 \mathrm{mg}$, and eventually with a therapeutic dose (up to $500 \mathrm{mg}$, depending on weight).

\section{STATISTICAL ANALYSIS}

The differences between percentages were evaluated with the $\chi^{2}$ test.

\section{Results}

We examined a total of 82 children ( 39 boys, 43 girls) ranging in age from 3 to 12 years (mean (SD) age: 6.8 (2.9) years) with histories of adverse cutaneous reactions to aminopenicillins. In over $90 \%$ of the cases, the antibiotic had been prescribed to treat upper respiratory tract infections. Our investigation was performed at a mean (SD) 30.6 (28.4) months (range 1-120) after the most recent drug exposure.

Sixty one of the 82 children $(74.4 \%)$ had reportedly reacted to amoxycillin (in three cases administered with clavulanic acid), 17 $(20.7 \%)$ had reacted to ampicillin (bacampicillin in one case, ampicillin plus sulbactam in another), and four had experienced reactions to more than one aminopenicillin (ampicillin and amoxycillin in three cases, ampicillin and bacampicillin in one). Thirteen (15.85\%) had also reacted to cephalosporins.

The reactive manifestations (based on parents' descriptions and, in some cases, paediatricians' reports) are summarised in table 1 . In $81 / 82$ cases, the symptoms had been exclusively cutaneous; the remaining child had experienced both cutaneous and respiratory symptoms. Forty two of the children had suffered maculopapular reactions, 36 had experienced urticaria/angio-oedema, and four had developed diffuse erythematous reactions.

On the basis of these tests, four of the 82 children $(4.9 \%)$ were diagnosed as having immediate hypersensitivity. All four had experienced their most recent reaction less than one year before testing. Two had experienced urticarial reactions, one had developed angiooedema, and the fourth had reacted with urticaria, angio-oedema, and dyspnoea. In all four cases, the onset of symptoms had occurred within one hour of the initial administration of the drug and the rash had persisted for approximately 24 hours after the antibiotic had been withdrawn and antihistaminic and steroid treatment started. Two of these four children presented skin test positivity for all of the allergens tested, the third was positive for PPL and penicillin $G$, and the fourth reacted only to penicillin G. RASTs were negative in 3/4; the fourth was positive for penicilloyls $G$ and $V$.

Thirty six other children with histories of urticarial/angio-oedematous or diffuse erythematous reactions appearing within 24 hours after the start of aminopenicillin treatment were negative in all tests. In 11 of these cases the most recent reaction had occurred less than one year before testing. Oral challenges were performed (nine with amoxycillin, two with ampicillin), none of which provoked symptoms.

Four other children (4.9\%) presented results indicative of cell mediated or delayed hypersensitivity: all had patch test positivity and late intradermal reactions to both ampicillin and amoxycillin; there were no delayed reactions to PPL, penicillin G, or MDM. All four had experienced morbilliform rashes, in one case accompanied by angio-oedema. The symptoms had appeared 24 hours or more after the start of aminopenicillin therapy and had persisted for 7-15 days after the drug had been discontinued and steroids had been started. The causes of previous reactions had been ampicillin in two cases (one of whom had experienced reactions during two separate 
cycles of treatment), bacampicillin in another, and amoxycillin in the fourth. The interval that had elapsed between the most recent reaction and our work-up was two years in two cases, one month in the third, and six years in the fourth.

The remaining 38 children who had experienced morbilliform rashes were negative in all allergologic tests. Oral challenges were carried out (34 with amoxycillin, four with ampicillin), none of which provoked symptoms.

\section{Discussion}

Our findings indicate that maculopapular rashes are common during aminopenicillin treatment, particularly among children. Roughly one third of the adults (60/195, $30.8 \%$ ) we examined recently in an analogous study ${ }^{12}$ developed them as opposed to a significantly higher percentage $(51.2 \%)$ among the 82 children described here $\left(\chi^{2}=9.52\right.$; $\mathrm{p}<0.01)$. Although maculopapular rashes are common in both allergic conditions and systemic infections, those occurring during antibiotic therapy are almost always attributed to the drug rather than to the infectious agent. Moreover, late (that is six hours or more) positive reactions to intradermal tests with the suspected drug (particularly those associated with patch test positivity for the same drug) are now considered indicative of delayed hypersensitivity. ${ }^{9-13}$ While this finding was quite common among our adults who reported morbilliform 'reactions' to aminopenicillins (33/60,55.0\%), only $9.5 \%$ of such reactions experienced by the children described here seemed to be cell mediated $\left(\chi^{2}=20.18 ; p<0.001\right)$. It would thus appear that maculopapular rashes developing in a child after several days of aminopenicillin treatment are much less likely to be manifestations of drug allergy than those reported by adults.

None the less, the possibility of an immune mediated reaction should be excluded by means of skin and patch testing, even in children. These tests must be performed with the aminopenicillin suspected of having caused the reaction, rather than with the major and minor penicillin determinants alone. Voorhorst and Sparreboom, in fact, found that the amino group of the aminopenicillin side chain is essential for a delayed allergic reaction to this group of drugs. ${ }^{16}$ Moreover, recent data indicate that $\mathrm{T}$ cell recognition of penicillins can be influenced by the side chain structure of the drug molecule. ${ }^{17}$ Failure to include the suspected aminopenicillin in the panel of test reagents might thus explain the negative allergologic findings reported in some patients who have reacted to these drugs. ${ }^{18}$

Our experience indicates that delayed hypersensitivity to aminopenicillins is a persistent condition. Therefore, negative results in patch and intradermal tests using an appropriate antigen panel appear to be a reliable indicator that the morbilliform rashes experienced by the patient are not immunological reactions to the drug, but rather manifestations of the underlying disease. In addition to those associated with the classic childhood exanthems, a number of other viruses that provoke non-specific upper respiratory tract symptoms can also produce morbilliform rashes. ${ }^{19}$ The underlying mechanisms are unclear in many cases: viral exanthems have been attributed to the destruction of infected epidermal cells by cytotoxic CD8 T cells, ${ }^{20}$ but they may also be the result of interaction between the virus and the antibiotic, as described in Epstein-Barr viral infections. ${ }^{21}$ On the other hand, some authors have reported positive reactions to oral challenges in cases of delayed rashes associated with $\beta$-lactam therapy in which skin tests were completely negative, even at the 48 hour reading. ${ }^{9}{ }^{13}$ In the light of these experiences (which differ from our own), it would appear that mechanisms unrelated to lymphocytes cannot be excluded. It should be emphasised that the RAST was negative for all 42 patients with maculopapular reactions. This result confirms our findings with adults and lead us to believe that this test could be omitted from the study protocol of such reactions.

Urticarial episodes during aminopenicillin therapy for an acute febrile illness may well be a direct effect of the infectious organism (for example Epstein-Barr virus, coxsackie viruses A9, A16, B4 and B5, echovirus 11 , etc) ${ }^{19}$ They may, of course, also be allergic manifestations, and in these cases appear to be typical of type I reactions.

Our experience indicates that skin test positivity for PPL, MDM, and/or penicillin $G$ is much more common in patients with immediate hypersensitivity to $\beta$-lactams than in those with the cell mediated form. In particular, all four children with IgE mediated sensitivity were penicillin $G$ positive, while none of those with delayed hypersensitivity was. However, as we observed in adults with immediate reactions, ${ }^{12}$ there may be cases of side chain specific hypersensitivity. Thus, until we have studies based on larger samples, we recommend using the entire panel of reagents.

Although the RAST proved to be less sensitive than the skin tests, we still recommend that it be performed in immediate reactions because, as we have observed with adults, there may be rare skin test negative, RAST positive cases. $^{12}$

The vast majority of the children in our study with urticarial reactions, however, presented negative skin test and RAST results. In such cases, some allergologists use oral challenges to confirm the absence of allergy. In a study of 346 skin test negative patients with histories of reactions to $\beta$-lactams, $3 \%$ reacted to penicillin challenges. ${ }^{2}$ Mendelson et al administered a 10 day oral penicillin challenge and repeated skin tests after four weeks or more. ${ }^{4}$ In a group of 219 patients (aged 4 months -20 years) subjected to this protocol, three developed self limiting skin symptoms during the challenge, but when repeated, skin tests were again negative. None of the other 216 experienced symptoms during the challenge, but two had positive skin tests after challenge.

In patients who have reacted to penicillins with urticaria and/or angio-oedema, however, 


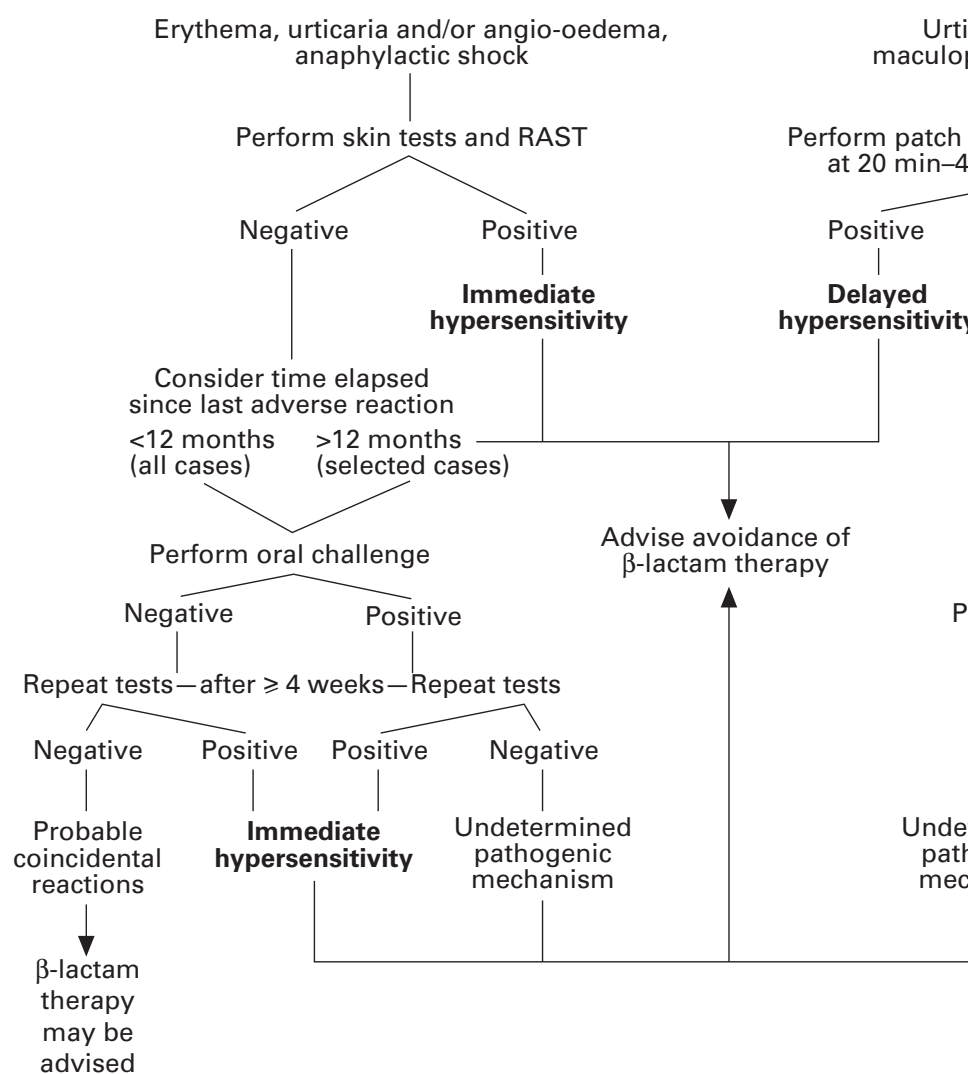

Urticarial or maculopapular rash

form patch tests and skin tests $20 \mathrm{~min}-48$ hour readings

Figure 1 Algorithm for allergologic work-up of adverse reactions to aminopenicillins.

negative skin test results must be interpreted in the light of the period that has elapsed since the last known exposure to the drug. In these cases, the frequency of positive skin test results declines as the interval between drug exposure and testing increases. ${ }^{2} 32223$ In a follow up study of 42 children with documented IgE mediated hypersensitivity to penicillins, Chandra et al observed the negativisation of skin tests in one third of the children who had presented positive reactions one year earlier. ${ }^{3}$

In the present study, of the 40 children who had experienced erythematous or urticarial reactions, only $15(37.5 \%)$ were tested by our staff within one year of their most recent reaction, including the four who presented skin tests indicative of IgE mediated sensitivity. In the remaining 11 cases, skin test negativity should be considered a fairly reliable indicator of the absence of immediate hypersensitivity, as confirmed in all cases by the absence of reactions to oral challenges.

In contrast, the test results are much less reliable for the 25 children who had negative skin tests more than one year after their urticarial/angio-oedematous reactions, and the symptoms experienced by a small percentage of them may have actually been manifestations of $\mathrm{IgE}$ mediated hypersensitivity. In these cases, considering parental resistance to these procedures and the risk of triggering another reaction, we generally defer oral challenges and advise avoidance of $\beta$-lactam treatment. In children who did not suffer severe reactions, for whom there is a greater than normal likelihood that penicillin therapy will be necessary, oral challenge confirmation of skin test negativity may be more appropriate. Taking into account the experiences of others as well as our own, we propose the diagnostic algorithm displayed in fig 1.

In any case, the testing protocol used here allowed us to clarify the nature of the 'reactions' experienced by $57(69.5 \%)$ of the 82 children studied. Hypersensitivity was confirmed in $9.8 \%$ of them. In 49 other children $(59.8 \%)$, the negative results of skin and patch testing as well as oral challenges allowed us to reasonably exclude the possibility of allergy to penicillins.

In conclusion, while the percentage of aminopenicillin associated skin rashes actually representing allergic phenomena is significantly lower in children than in adults, our study demonstrates that the possibility of both immediate and delayed hypersensitivity does exist. The diagnostic protocol described here can be helpful in clarifying the nature of many of these reactions. Any doubt can be eliminated with controlled oral challenges and, if necessary, retesting.

\footnotetext{
1 Saxon A. Immediate hypersensitivity reactions to betalactam antibiotics. Ann Intern Med 1987;107:204-15.

2 Green GR, Rosenblum AH, Sweet LC. Evaluation of penicillin hypersensitivity: value of clinical history and skin test-
} 
ing with penicilloyl-polylysine and penicillin G. F Allergy Clin Immunol 1977;60:339-45.

3 Chandra RK, Joglekar SA, Tomas E. Penicillin allergy: antipenicillin IgE antibodies and immediate hypersensitivity skin reactions employing major and minor determinants of penicillin. Arch Dis Child 1980;55:857-60

4 Mendelson LM, Ressler C, Rosen JP, Selcow JE. Routine elective penicillin allergy skin testing in children and adolescents: study of sensitisation. $\mathcal{F}$ Allergy Clin Immunol 1984;73:76-81.

5 Shapiro S, Siskind V, Slone D, Lewis GP, Jick H. Drug rash with ampicillin and other penicillins. Lancet 1969;ii:96972 .

6 Collaborative Study Group. Prospective study of ampicillin rash. BMF 1973;i:7-9.

7 Kerns DL, Shira JE, Go S, et al. Ampicillin rash in children. Relationship to penicillin allergy and infectious mononucleosis. Am 7 Dis Child 1973;125:187-90.

8 de Haan P, Bruynzeel DP, Van Ketel WG. Onset of penicillin rashes: relation between type of penicillin administered lin rashes: relation between type of penicilin administerc

9 Aihara M, Ikezawa Z. Evaluation of the skin test reactions with delayed type rash induced by penicillins and cephalosporins. Dermatol 1987;14:440-8.

10 Vega JM, Blanca M, Carmona MJ, et al. Delayed allergic reactions to betalactams. Allergy 1991;46:154-7.

11 Warrington RJ, Silviu-Dan F, Magro C. Accelerated cell-mediated immune reactions in penicillin allergy. $\mathcal{F}$ Allergy Clin Immunol 1993;92:626-8.

12 Romano A, Di Fonso M, Papa G, et al. Evaluation of adverse cutaneous reactions to aminopenicillins with emphasis on those manifested by maculopapular rashes. Allergy 1995;50:113-8.
13 Terrados S, Blanca M, Garcia J, et al. Nonimmediate reactions to betalactams: prevalence and role of the different penicillins. Allergy 1995;50:563-7.

14 Bierman CW, Pierson WE, Zeitz SJ, Hoffman LS, VanArsdel PP Jr. Reactions associated with ampicillin therapy. FAMA 1972;220:1098-100

15 Wilkinson DS, Fregert S, Magnusson B, et al. Terminology of contact dermatitis. Acta Dermatovener 1970;50:287-92.

16 Voorhorst R, Sparreboom S. The use of stereoisomers in patch testing. Ann Allergy 1980;45:100-3.

17 Padovan E, Mauri-Hellweg D, Pichler WJ, Weltzien HU. T cell recognition of penicillin G: structural features determining antigenic specificity. Eur F Immunol 1996;26:42-8.

18 Graff-Lonnevig V, Hedlin G, Lindfors A. Penicillin allergy - a rare paediatric condition ? Arch Dis Child 1988; 63:1342-6.

19 Cherry JD. Cutaneous manifestations of systemic infections. In: Feigin RD, Cherry JD, eds. Textbook of pediatric infectious diseases. Philadelphia: WB Saunders, 1987: 786-817.

20 Chosidow O, Bourgault I, Roujeau JC. Drug rashes. What are the targets of cell-mediated cytotoxicity? Arch Dermatol 1994;130:627-9

21 Haverkos HV, Amsel Z. Drotman P. Adverse virus-drug interactions. Rev Infect Dis 1991;13:697-704.

22 Finke SR, Grieco MH, Connel JT, Smith EC, Sherman WB. Results of comparative skin tests with penicilloyl-polylysine and penicillin in patients with penicillin allergy. Am f Med 1965;38:71-82.

23 Sullivan TJ, Wedner HJ, Shatz JS, Yiecies LD, Parker CW. Skin testing to detect penicillin allergy. F Allergy Clin Immunol 1981;68:171 -80. 